\title{
A combined measurement of thermal and mechanical relaxation
}

\author{
Tage Christensen *, Bo Jakobsen, Jon Papini, Tina Hecksher, Jeppe C. Dyre, Niels Boye Olsen \\ DNRF Centre “Glass and Time”, IMFUFA, Department of Sciences, Roskilde University, Postbox 260, DK-4000 Roskilde, Denmark
}

\section{A R T I C L E I N F O}

\section{Article history:}

Received 18 April 2010

Received in revised form 29 June 2010

\section{Keywords:}

Glass transition;

Thermoviscoelasticity;

Prigogine-Defay ratio

\begin{abstract}
A B S T R A C T
In order to describe relaxation the thermodynamic coefficient $\frac{1}{\beta_{S}}=\left(\frac{\partial V}{\partial S}\right)$ can be generalized into a complex frequency-dependent cross response function. We explore theoretically the possibility of measuring $\frac{1}{\beta_{s}}(\omega)$ for a supercooled liquid near the glass transition. This is done by placing a thermistor in the middle of the liquid which itself is contained in a spherical piezoelectric shell. The piezoelectric voltage response to a thermal power generated in the thermistor is found to be proportional to $\frac{1}{\beta_{s}}(\omega)$ but factors pertaining to heat diffusion and adiabatic compressibility $\kappa_{S}(\omega)$ do also intervene. We estimate a measurable piezoelectric voltage of $1 \mathrm{mV}$ to be generated at $1 \mathrm{~Hz}$ for a heating power of $0.3 \mathrm{~mW}$. Together with $\kappa_{S}(\omega)$ and the longitudinal specific heat $c_{l}(\omega)$ which may also be found in the same setup a complete triple of thermoviscoelastic response functions may be determined when supplemented with shear modulus data.
\end{abstract}

(C) 2010 Elsevier B.V. All rights reserved.

\section{Introduction}

The recent finding [1] that a class of liquids - the strongly correlating liquids - may be described by a single "order" parameter makes it urgent to devise methods that measure thermal and mechanical relaxation and their interconnection. It would be an advantage if they can be measured in the same setup on the same sample. The classical Prigogine-Defay test of a one "order" parameter description has recently been rigorously reformulated for the equilibrium liquid in terms of (four) Dynamic Prigogine-Defay ratios [2]. One of these, $\Lambda_{S p}=-\left(T_{0} / c_{p}\right)^{\prime \prime}\left(\kappa_{S}\right)^{\prime \prime} /\left(\left(1 / \beta_{S}\right)^{\prime \prime}\right)^{2}$ is from an experimental viewpoint the easiest to access. It contains the complex frequencydependent specific heat $c_{p}(\omega)$, adiabatic compressibility $\kappa_{S}(\omega)$ and adiabatic pressure coefficient $\beta_{S}(\omega) \equiv(\delta p(\omega) / \delta T(\omega))_{s}$. We can measure $\kappa_{S}(\omega)$ by the so-called piezoelectric bulk modulus gauge (PBG) [3]. The PBG is a hollow sphere with a thin wall of a piezoelectric ceramic material. Pressure/volume changes of a contained liquid are detectable due to the piezoelectric effect. In the middle of the PBG we have now added a thermistor by which we can measure the longitudinal heat capacity $c_{l}(\omega)$ via the effusivity $[4,5]$. In this paper we study theoretically what can be deduced by combining the two sensors, i.e. how does the expansion of the liquid upon heating in the centre affect the piezoelectric shell.

\section{Thermomechanical response of a differential volume element}

The thermal interaction with matter is described in terms of the conjugated variables temperature, $T$ and entropy, $S$. We name the interaction as an energy bond. It is a scalar bond since the variables are scalars. The mechanical interaction is described in terms of the strain and stress tensors but this interaction can be separated in a pure scalar part by the trace of these tensors and the deviatoric traceless part of these tensors. The conjugated variables of the scalar mechanical energy bond may then be taken as volume, $V$ and minus pressure, $-p$. The deviatoric parts of the strain and stress tensors describe shear deformations and are not coupled to the scalar parts for symmetry reasons (The Curie-Prigogine principle [6-8]) but the scalar bonds however are coupled. The response $\delta S$ and $\delta V$ to perturbations $\delta T$ and $-\delta p$ defines the constitutive properties of matter:

$d V / V_{0}=-\kappa_{T} d p+\alpha_{p} d T$

$d S / V_{0}=-\alpha_{p} d p+\frac{1}{T_{0}} c_{p} d T$

Since the perturbations excite thermal and acoustical waves the constitutive equations are defined for a differential volume element, $V_{0}$ of a linear dimension, $R$ much smaller than the characteristic thermal diffusion length and acoustical wave length associated with the time scale of the perturbations (Figs. 1 and 2).

Eqs. (1) and (2) are valid in equilibrium thermodynamics. When it comes to describing the relaxation of supercooled liquids they are replaced with corresponding equations of linear irreversible thermodynamics

\footnotetext{
* Corresponding author.

E-mail address: tec@ruc.dk (T. Christensen).
} 




Fig. 1. The two scalar energetic interactions with a differential volume element. Differential means that the wavelengths of the thermal and mechanical perturbations are much longer than the dimensions of the volume element.

$d S(t) / V_{0}=-\int_{-\infty}^{t} \alpha_{p}\left(t-t^{\prime}\right) d p\left(t^{\prime}\right)+\int_{-\infty}^{t} \frac{1}{T_{0}} c_{p}\left(t-t^{\prime}\right) d T\left(t^{\prime}\right)$

The thermodynamic coefficients are now replaced by response functions. These relaxing response functions may be consider in the frequency domain instead by defining e.g. the complex frequencydependent compressibility as:

$\kappa_{T}(\omega)=i \omega \int_{0}^{\infty} \kappa_{T}(t) e^{-i \omega t} d t$

Now $d V, d S, d p$ and $d T$ should be interpreted as the complex amplitudes of harmonically varying perturbations and the constitutive equations of linear irreversible thermodynamics (3) and (4) becomes

$d V / V_{0}=-\kappa_{T}(\omega) d p+\alpha_{p}(\omega) d T$

$d S / V_{0}=-\alpha_{p}(\omega) d p+\frac{1}{T_{0}} c_{p}(\omega) d T$

They can now be treated exactly like the equilibrium Eqs. (1) and (2). The response functions like $\kappa_{T}(\omega)$ and $c_{p}(\omega) / T_{0}$ pertaining to the conjugated variables of a single energy bond are auto response functions. $\alpha_{p}(\omega)$ on the other hand is a cross response function connecting a variable from the thermal bond to a variable from the mechanical bond. The three functions give a complete description of the thermomechanical response. For relaxing system they are not completely independent since the knowledge of the cross response function and one of the auto response functions for all frequencies makes it possible to calculate the other auto response function $[9,10]$. Moreover if the liquid relaxation is described by a single order parameter the relaxational part of the triple of relaxation functions are proportional and the dynamic Prigogine-Defay ratio [2]

$\Lambda_{T p}=\frac{c_{p}^{\prime \prime} \kappa_{T}^{\prime \prime}}{T_{0}\left(\alpha_{p}^{\prime \prime}\right)^{2}}$

is equal to 1 .

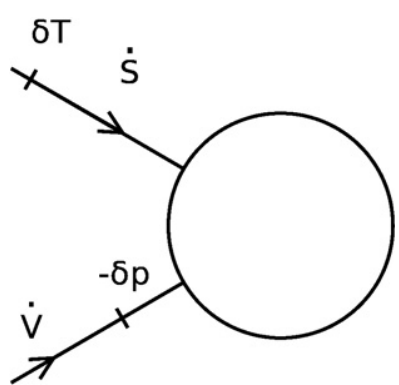

Fig. 2. Another response situation. Here entropy and pressure are the input variables and marked on the energy bond nearest to the system. In Fig. (1) temperature and pressure were the input variables.
There are three other different possibilities of pairs of independent controlling variables than $(d T,-d p)$, namely $(d S, d V),(d S,-d p),(d T, d V)$ leading to other triples of response functions and other variants of the dynamic Prigogine-Defay ratio. It is thus convenient to introduce the four auto response functions (connecting conjugated variables of the same bond),

$c_{V}=\frac{T}{V}\left(\frac{\partial S}{\partial T}\right)_{V}, \quad c_{p}=\frac{T}{V}\left(\frac{\partial S}{\partial T}\right)_{p}$

$\kappa_{T}=-\frac{1}{V}\left(\frac{\partial V}{\partial p}\right)_{T}, \quad \kappa_{S}=-\frac{1}{V}\left(\frac{\partial V}{\partial p}\right)_{S}$

and the four cross response functions (connecting variables of different bonds),

$\alpha_{p}=\frac{1}{V}\left(\frac{\partial V}{\partial T}\right)_{p}=-\frac{1}{V}\left(\frac{\partial S}{\partial p}\right)_{T}$

$\frac{1}{\alpha_{S}}=-V\left(\frac{\partial T}{\partial V}\right)_{S}=V\left(\frac{\partial p}{\partial S}\right)_{V}$,

$\beta_{V}=\left(\frac{\partial p}{\partial T}\right)_{V}=\left(\frac{\partial S}{\partial V}\right)_{T}$

$\frac{1}{\beta_{S}}=\left(\frac{\partial T}{\partial p}\right)_{S}=\left(\frac{\partial V}{\partial S}\right)_{p}$

Strictly speaking - defining these 8 functions as partial derivatives they are at first just constant real thermodynamic coefficients but they may be generalized into complex functions just like $\kappa_{T}(\omega), \alpha_{p}(\omega)$ and $c_{p}(\omega)$ and they are thought of in this sense in the following. An extensive table of relations between these functions is given in the appendix of reference [4]. Here we just notice that $\beta_{S}$ is related to $\alpha_{p}$ and $c_{p}$ by

$\frac{1}{T_{0} \beta_{S}}=\frac{\alpha_{p}}{c_{p}}$

All of the response functions can be related to fluctuations of the thermodynamic variables [11]. For example $1 / \beta_{S}$ is proportional to correlations between temperature and volume fluctuations. It was recently found [1] that a class of liquids - the strongly correlating liquids - may be described by a single "order" parameter and it was explicitly shown [12] by computer NVT simulations of the KobAndersen binary Lennard-Jones system that

$\Lambda_{T V}(\omega)=-\frac{c_{V}^{\prime \prime}\left(\frac{1}{\kappa_{T}}\right)^{\prime \prime}}{T_{0}\left(\beta_{V}^{\prime \prime}\right)^{2}}$

was 1 within $20 \%$.

As we shall see it will probably be the triple $T_{0} / c_{p}(\omega), \kappa_{S}(\omega), 1 / \beta_{S}$ that is experimentally easiest accessible and it will be the $S p$-variant of the Prigogine-Defay ratio

$\Lambda_{S p}=-\frac{\left(\frac{T_{0}}{c_{p}}\right)^{\prime \prime} \kappa_{S}^{\prime \prime}}{\left(\left(\frac{1}{\beta_{S}}\right)^{\prime \prime}\right)^{2}}$

that shall test the one-parameter'ness of real liquids.

\section{Thermomechanical response of a finite spherical volume element}

When considering a real experiment with perturbations varying at a frequency $f=\omega /(2 \pi)$ it is not always possible to be in a situation of homogeneous fields. The wavelength of sound $\lambda_{\text {sound }}$ and the heat diffusion length, $\left|l_{D}\right|$ may be comparable to or smaller than the sample size $R$. If we consider frequencies below $1 \mathrm{kHz}$ then roughly $\lambda_{\text {sound }}>1 \mathrm{~m}$ 
and for $R<1 \mathrm{~cm}$ we can neglect mechanical waves i.e. neglect inertia in the continuum description [4]. However the heat diffusion length, $\left|l_{D}\right|=|\sqrt{D /(i \omega)}|$ of a supercooled liquid with a typical heat diffusion constant of $D=0.1 \mathrm{~mm}^{2} / \mathrm{s}$ varies from $4 \mu \mathrm{m}$ to $4 \mathrm{~mm}$ when frequency varies from $1 \mathrm{kHz}$ to $1 \mathrm{mHz}$ and thus heat diffusion cannot be neglected for a sample size of $1 \mathrm{~cm}$. By the coupling between the temperature field and the strain field that $\alpha_{p}$ induces, the strain and stress fields also become inhomogeneous. This implies that even in spherical geometry the two pressures, the radial $\delta p_{r}=-\sigma_{r r}$ and the mean (hydrostatic) $\delta p=-1 / 3\left(\sigma_{r r}+\sigma_{\theta \theta}+\sigma_{\varphi \varphi}\right)$ are not equal if shear modulus is comparable to bulk modulus. When interacting mechanically with a sphere through its surface we don't have access to $\delta p$ but only to $\delta p_{r}$. For this reason shear modulus enters - via the boundary conditions - the description of the thermomechanical response of a finite sphere although it wasn't present in the thermomechanical response of a differential volume element, Eqs. (6) and (7). Consider generally a finite amount of liquid lying in between radii $r_{1}$ and $r_{2}$ depicted in Fig. 3 . In the inertia-free limit the general problem of the relation between the variables, radial pressure, $\delta p_{r}$, temperature change, $\delta T$, volume displacement, $\delta V$ and entropy displacement, $\delta S$ at the two radii has been solved [4] in the frequency domain in terms of a transfer matrix:

$$
\left(\begin{array}{c}
\delta p_{r} \\
\delta T \\
\delta V \\
\delta S
\end{array}\right)_{r_{2}}=\mathbf{T}\left(r_{2}, r_{1}\right)\left(\begin{array}{c}
\delta p_{r} \\
\delta T \\
\delta V \\
\delta S
\end{array}\right)_{r_{1}}
$$

In general $\mathbf{T}$ is a complicated object. An interesting result was found when two conditions hold: 1) frequencies are high enough to be in the "thermally thick limit" with respect to $r_{2}$, i.e. $\left|l_{D}\right| \ll r_{2}$ and 2) $r_{1} \ll r_{2}$ : When studying in this case the combined response to thermal stimuli at radius $r_{1}$ and mechanical stimuli at radius $r_{2}$ one can neglect the mechanical boundary condition at $r_{1}$ and the thermal boundary condition at radius $r_{2}$ ending up with a reduced transfer matrix given as

$$
\left(\begin{array}{c}
\delta T \\
\delta S
\end{array}\right)_{r_{1}}=\left(\begin{array}{cc}
i \omega Z_{\text {th }} T_{0} V_{2} \kappa_{S} \beta_{S} & i \omega Z_{\text {th }} T_{0} \beta_{S} \\
V_{2} \kappa_{S} \beta_{S} & \beta_{S}
\end{array}\right)\left(\begin{array}{c}
\delta p_{r} \\
\delta V
\end{array}\right)_{r_{2}},
$$

where $V_{2}=\frac{4 \pi}{3} r_{2}^{3}$ and $Z_{\mathrm{th}}$ is the thermal impedance,

$$
Z_{\mathrm{th}}(\omega)=\frac{1}{4 \pi \lambda r_{1}\left(1+\sqrt{i \omega r_{1}^{2} c_{l}(\omega) / \lambda}\right)},
$$

$\lambda$ is the heat conductivity. The specific heat, $c_{l}$ entering the thermal impedance is the so-called longitudinal specific heat. $c_{l}$ is the amount of heat absorbed per Kelvin upon a temperature increment if the associated expansion is forced to be longitudinal. This is in contrast to the isobaric specific heat for which the expansion is isotropic. The

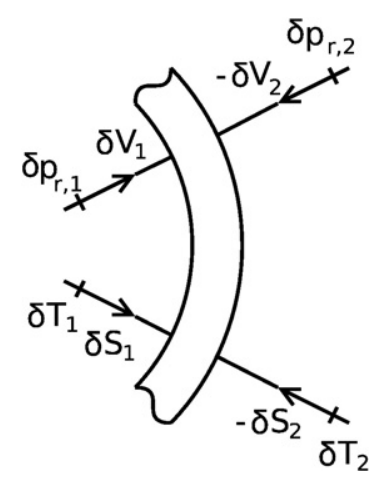

Fig. 3. Depiction of the four thermal and mechanical interactions at the boundaries at $r_{1}$ and $r_{2}$ in spherical geometry. longitudinal specific heat can be related to the isochoric specific heat, $c_{V}$ by [4]

$c_{l}=\frac{\frac{1}{\kappa_{S}}+\frac{4}{3} G}{\frac{1}{\kappa_{T}}+\frac{4}{3} G} c_{V}$,

where $G$ is shear modulus. Using the identities [4]

$\frac{c_{p}}{c_{V}}=\frac{\kappa_{T}}{\kappa_{S}}$ and $\kappa_{T}-\kappa_{S}=\frac{c_{p}}{T_{0} \beta_{S}^{2}}$

together with (15) the deviation between the longitudinal specific heat and isobaric specific heat may be expressed by

$\frac{1}{c_{p}}=\frac{1}{c_{l}}-\frac{1}{T_{0} \beta_{S}^{2}} \frac{\frac{4}{3} G}{1+\frac{4}{3} G \kappa_{S}}$.

This expression has the advantage of giving $c_{p}(\omega)$ in terms of the quantities $c_{l}(\omega), \kappa_{S}(\omega), \beta_{S}(\omega)$ and $G(\omega)$ that are possible to access experimentally by our new device supplemented with the Piezoelectric Shear modulus Gauge [13].

Eq. (13) is equivalent to equation (138) of reference [4]. The determinant of (13) is zero although a transfer matrix relating proper conjugated variables should have determinant 1 . The reason is that we are studying a limiting case where $\left|i \omega Z_{\mathrm{th}} T_{0} V_{2} \kappa_{S} \beta_{S}^{2}\right| \gg 1$. Thus the inverse relation is

$\left(\begin{array}{c}\delta p_{r} \\ \delta V\end{array}\right)_{r_{2}}=\left(\begin{array}{cc}\beta_{S} & -i \omega Z_{t h} T_{0} \beta_{S} \\ -V_{2} \kappa_{S} \beta_{S} & i \omega Z_{t h} T_{0} V_{2} \kappa_{S} \beta_{S}\end{array}\right)\left(\begin{array}{c}\delta T \\ \delta S\end{array}\right)_{r_{1}}$,

This is equivalent to equation (139) of reference [4], but there was a typo: the common $T_{0}$ factor in the matrix of that formula should be deleted. The simplified transfer matrix can be represented by the equivalent diagram of Fig. 4 . The equivalent diagram is in a sense a more correct description since it leads to a transfer matrix deviating from Eq. (13) by a negligible term that however endows it with a determinant of 1 .

\section{The combined experiment}

The adiabatic compressibility $\kappa_{S}(\omega)$ can be measured using the piezoelectric bulk modulus gauge (PBG) [3]. The PBG is a hollow sphere of radius $1 \mathrm{~cm}$ with a thin wall of a piezoelectric ceramic material. The thickness $t$ is $0.5 \mathrm{~mm}$. The sphere may be filled by a liquid at elevated temperature, where it is fluent. The PBG transforms the mechanical compliance of the liquid into an electric compliance (the capacitance), that can be simply measured by an LCR-meter or by other means. In order to make combined thermomechanical experiments we have placed a thermistor in the middle of the PBG (see Fig. 5). By the thermistor itself we can measure the longitudinal heat capacity $c_{l}(\omega)$ via the effusivity [5]. Combining the two devices makes it, in principle, possible to get the cross response function $1 / \beta_{s}$. That is, nearly all ingredients of $\Lambda_{s p}$ can be found for the same sample in the same device. However if $c_{l}(\omega)$ differs significantly from $c_{p}(\omega)[4]$ as

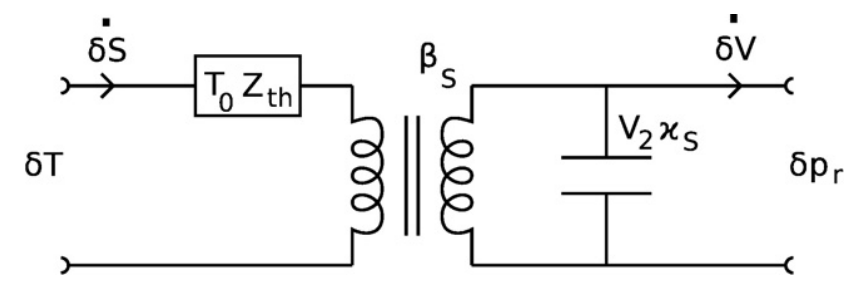

Fig. 4. Equivalent diagram of the liquid. 


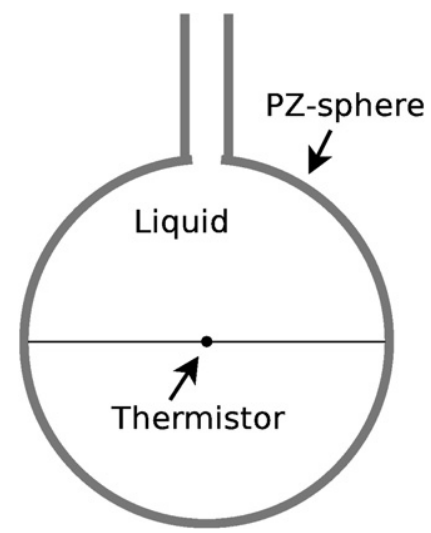

Fig. 5. The combined measurement of $c_{l}, \kappa_{S}, \beta_{S}$.

may be judged by Eq. (17) a supplementary measurement of the shear modulus is needed. We may produce an oscillating heat current with amplitude $P_{\text {th }}$ by Joule heating in the thermistor and measure the piezoelectric voltage $U_{p z}$ generated in the PBG as the liquid attempts to expand. This voltage contain information on $1 / \beta_{S}=\left(\frac{\partial V}{\partial S}\right)_{p}$ but it is also dependent of the thermal interaction of the thermistor with the liquid and the mechanical interaction of the liquid with the PBG. In order to filter these factors out we may look at the equivalent diagram, Fig. 6 of the whole system. For simplicity we model the thermistor as an ideal heat generator in parallel with its heat capacitance $C_{0}$ of approximately $5.5 \times 10^{-5} \mathrm{~J} / \mathrm{K}$. (For a more detailed model of the thermal structure of the thermistor, see reference [5]). In the equivalence diagram in Fig. 6 the PBG consist of a mechanical compliance, $C_{m}$, a transducer ratio, $T_{p z}$ and an electric (clamped) capacitance $C_{e}$. They can be expressed [3] in terms of the dielectric constant, $\epsilon_{33}$, the elastic compliance, $\left(s_{11}+s_{12}\right) / 2$ and the piezoelectric constant, $d_{13}$ of the piezoelectric material pz29 together with the radius, $r_{2}$ and shell thickness, $t$ (see Table 1 ).

By the equivalence diagram one finds that the generated piezoelectric voltage amplitude $U_{p z}$ measured by a voltmeter of high impedance $\left(I_{p z}=0\right)$ in response to a heat current amplitude $P_{t h}$ generated in the thermistor becomes

$$
\begin{aligned}
\left(\frac{U_{p z}}{P_{t h}}\right)_{I_{p z}=0}= & \frac{T_{p z}}{C_{e} i \omega} \frac{1}{\left(1+C_{0} i \omega Z_{t h}(\omega)\right)\left(1+\left(1+T_{p z}^{2} \frac{C_{m}}{C_{e}}\right) \frac{V_{2} \kappa_{S}(\omega)}{C_{m}}\right)} \\
& \frac{1}{T_{0} \beta_{S}(\omega)}
\end{aligned}
$$

We see that in principle $\beta_{S}$ may be found by this third cross experiment with a thermistor in the PBG. However the signal is also influenced in its frequency dependence by the thermal impedance of the liquid and the adiabatic compressibility but both of these can be found by the experiments of the thermistor alone respectively the
Table 1

Properties and lumped parameters of the piezoelectric bulk modulus gauge modeled in Fig. 6.

\begin{tabular}{lll}
\hline$r_{2}$ & & $9.5 \times 10^{-3} \mathrm{~m}$ \\
$t$ & & $0.5 \times 10^{-3} \mathrm{~m}$ \\
$\epsilon_{33} s_{11}+s_{12}$ & & $26 \times 10^{-9} \mathrm{~F} / \mathrm{m}$ \\
$d_{13}{ }^{2}$ & & $6 \times 10^{-12} \mathrm{~m}^{2} / \mathrm{N}$ \\
$k_{p}$ & $\frac{d_{13}}{\sqrt{\epsilon_{33}\left(s_{11}+s_{12}\right) / 2}}$ & $26 \times 10^{-9} \mathrm{C} / \mathrm{N}$ \\
$C_{m}$ & $\frac{s_{11}+s_{12}}{2} \frac{4 \pi r_{2}^{4}}{t}$ & 0.64 \\
$T_{p z}$ & $\frac{2 d_{13}}{\left(s_{11}+s_{12}\right)} \frac{1}{r_{2}}$ & $1.5 \times 10^{-15} \mathrm{~m}^{3} / \mathrm{Pa}$ \\
$C_{e}$ & $\epsilon_{33}\left(1-k_{p}^{2}\right) \frac{4 \pi r_{2}^{3}}{t}$ & $4 \times 10^{3} \mathrm{C} / \mathrm{m}^{3}$ \\
& & $40 \times 10^{-9} \mathrm{~F}$
\end{tabular}

PBG alone. The frequency dependence in the thermal impedance has a characteristic diffusion time constant that is almost independent of the change of $c_{l}$ at the glass transition whereas the factor containing the compressibility of course will change the position of its characteristic time scale as temperature is changed. It is interesting to estimate this signal. At $1 \mathrm{~Hz} C_{0} Z_{t h}$ is of the order of 1 and so is the factor containing the compressibility. From the values in the Table 1 we find $\frac{T_{p z}}{C_{e}}=10^{11} \mathrm{Vm}^{-3}$. Typical values of the expansion coefficient and the specific heat of a liquid are $\alpha_{p}=5 \times 10^{-4} \mathrm{~K}^{-1}$ and $c_{p}=2 \times$ $10^{6} \mathrm{JK}^{-1} \mathrm{~m}^{-3}$ and thus $\frac{1}{T_{0} \beta_{S}}=\frac{\alpha_{p}}{c_{p}}=2.5 \times 10^{-10} \mathrm{~m}^{3} \mathrm{~J}^{-1}$. From this we find $\frac{T_{p z}}{C_{e}} \frac{1}{T_{0} \beta_{S}}=25 \mathrm{~V} / \mathrm{J}$. Using a power amplitude $P_{t h}$ of $0.3 \mathrm{~mW}$ in order to keep temperature change in the centre below $1 \mathrm{~K}$ we thus expect a signal of the order of $1 \mathrm{mV}$ at $1 \mathrm{~Hz}$ which is readily detectable.

\section{Conclusion}

Of the four dynamic Prigogine-Defay ratios one special namely, $\Lambda_{s p}=-\left(T_{0} / c_{p}\right)^{\prime \prime}\left(\kappa_{S}\right)^{\prime \prime} /\left(\left(1 / \beta_{S}\right)^{\prime \prime}\right)^{2}$ seems from an experimental viewpoint to be the most directly accessible. By combining the devices of the two techniques 1 ) measurement of the adiabatic compressibility $\kappa_{S}(\omega)$ with the Piezoelectric Bulk modulus Gauge and 2) measurement of the longitudinal specific heat $c_{l}(\omega)$ by thermal effusion in spherical geometry a third cross response function, $1 / \beta_{S}(\omega)$ may be measured. That is, nearly all ingredients of $\Lambda_{s p}$ could be found for the same sample in the same device. However $c_{l}(\omega)$ may differ from $c_{p}(\omega)$ [4], in which case a supplementary measurement of the shear modulus is needed.

\section{References}

[1] U.R. Pedersen, T. Christensen, T. Schrøder, J.C. Dyre, Feasibility of a singleparameter description of equilibrium viscous liquid dynamics, Phys. Rev. E 77 (2008) 011201.

[2] N.L. Ellegaard, T. Christensen, P.V. Christiansen, N.B. Olsen, U.R. Pedersen, T.B. Schrøder, J.C. Dyre, Single-order-parameter description of glass-forming liquids: a one-frequency test, J. Chem. Phys. 126 (2007) 074502.



Fig. 6. Equivalent diagram of the liquid in contact with the thermistor and the piezoelectric shell (PBG). 
[3] T. Christensen, N.B. Olsen, Determination of the frequency-dependent bulk modulus of glycerol using a piezoelectric spherical-shell, Phys. Rev. B 49 (1994) 15396.

[4] T. Christensen, J.C. Dyre, Solution of the spherically symmetric linear thermoviscoelatic problem in the inertia-free limit, Phys. Rev. E 78 (2008) 021501.

[5] B. Jakobsen, N.B. Olsen, T. Christensen, Frequency dependent specific heat from thermal effusion in spherical geometry, Phys. Rev. E 81 (2010) 061505.

[6] P. Curie, Oeuvres, Societe Francaise de Physique, Paris, 1908.

[7] I. Prigogine, Etude Thermodynamique des Phenomenes Irreversibles, Desoer, Liege, 1947.

[8] S.R.D. Groot, P. Mazur, Non-equilibrium Thermodynamics, North-Holland Publishing Co., Amsterdam, 1962.

[9] N.P. Bailey, T. Christensen, B. Jakobsen, K. Niss, N.B. Olsen, U.R. Pedersen, T.B. Schrøder, J.C. Dyre, Glass-forming liquids: one or more "order" parameters? J. Phys. Condens. Matter 20 (2008) 244113.
[10] J. Meixner, H.G. Reik, in: S. Flügge (Ed.), Principen der Thermodynamik und Statistik, Handbuch der Physik, vol. 3, Springer, Berlin, 1959.

[11] T.B. Schrøder, N.P. Bailey, U.R. Pedersen, N. Gnan, J.C. Dyre, Pressure-energy correlations in liquids. III. Statistical mechanics and thermodynamics of liquids with hidden scale invariance. J. Chem. Phys. 131 (2009) 234503.

[12] N.P. Bailey, U.R. Pedersen, N. Gnan, T.B. Schrøder, J.C. Dyre, Pressure-energy correlations in liquids. II. Analysis and consequences. J. Chem. Phys. 129 (2008) 184508.

[13] T. Christensen, N.B. Olsen, A rheometer for the measurement of a high-shear modulus covering more than 7 decades of frequency below $50 \mathrm{kHz}$, Rev. Sci. Instrum. 66 (1995) 5019-5031. 